and to try translated versions of the tool to maximise the participation of respondents with limited knowledge of English in a diverse ethnic population

Background Patient/parent satisfaction surveys are important monitoring tools used in the national health service (NHS). Before the Urgent-and-Emergency-Care PREM tool was published by the RCPCH in October 2012 there was no standard feedback form for paediatric A\&E. This new tool is a well researched and standardised tool for obtaining children/parent feedback.

Methods Demographic data from the census was collected which showed that our NHS trust caters for a population with a large percentage of Turkish/Greek Cypriot, African and Somalian ethnicity. There is an annual attendance of about 38000 to children's A\&E. While doing the survey, we eliminated the bias due to languagebarrier by translating the RCPCH tool in Turkish and Somalian, the two commonly spoken languages in our ethnic population-group. The PREM tool was translated by doctors with knowledge of the languages and was colour coded for adult or children versions. Questionnaires were given to consecutive willing parents and/or children while waiting in the department. The feedback forms were analysed on Microsoft-Excel using common statistical methods.

Results Total of 50 feedback forms were collected. Feedback was given by $12(24 \%)$ children, $29(64 \%)$ parents and $4(9 \%)$ by both. The study group had 19(41\%) European, 9(20\%) Asian, 12(26\%) African and $2(4 \%)$ mixed. The main languages spoken were English $25(57 \%)$, other European 10(22\%), and all other 10(22\%). The main highlights of the survey was that 43 of $46(94 \%)$ respondents were satisfied by the services, 24(49\%) waited longer than expected, 15(32\%) wanted better information while waiting and $4(8 \%)$ were not given adequate privacy.

Conclusions The introduction of feedback-forms in multiple languages has perhaps given a more unbiased feedback with more involvement of the ethnic subgroups. The results were overall satisfying but a few specific areas that need improvement were identified. The responses to individual questions will also serve as a baseline for serial monitoring after implementing changes and training in problem areas.

\section{G32 DEVELOPING PRINCIPLES FOR DELIVERING AND COMMISSIONING BETTER HEALTH OUTCOMES AND EXPERIENCES FOR CHILDREN AND YOUNG PEOPLE SO THEY ARE COMPARABLE WITH THE BEST IN THE WORLD}

doi:10.1136/archdischild-2013-304107.045
${ }^{1,2} \mathrm{M}$ Clements, ${ }^{1,3} \mathrm{~K}$ Evans, ${ }^{1} \mathrm{~T}$ Cogan, ${ }^{1,4} \mathrm{C}$ Upton, ${ }^{1,5} \mathrm{D}$ Williams, ${ }^{1,6} \mathrm{~S}$ Evans. ${ }^{1}$ Strategic Network for Child Health and Well-Being, NHS East of UK, Cambridge, UK; ${ }^{2}$ Department of Paediatrics, West Suffolk Hospital NHS Foundation Trust, Bury St Edmunds, UK; ${ }^{3}$ Children \& Young People's Programme, NHS Institute for Innovation and Improvement, Coventry, UK; ${ }^{4}$ Department of Paediatrics, Norfolk and Norwich Hospital NHS Foundation Trust, Norwich, UK; ${ }^{5}$ Department of Paediatrics, Addenbrookes Hospital NHS Foundation Trust, Cambridge, UK; ${ }^{6}$ Child Health, Eastern Region Public Health, Cambridge, UK

Aim The development of a single set of principles to be used by providers and commissioners, across the whole healthcare system wherever a child or young person is seen. The aim is to improve the health outcomes and experiences for children and young people (CYP).

Methods A multi-professional team of GPs, Health Visitors, School Nurses, Paediatricians, Public health, Children's Nurses, patient and family representatives came together over a period of 6 months in a number of facilitated work- shops.

CYP and family engagement was central to this work with visits to Children's Centres, primary and secondary schools to listen to families experiences of health services. A graphic illustrator captured the key messages from each engagement event.

The principles were referenced against the Children and Young People's Outcome Forum Report and the NHS Mandate.

Results A set of 6 principles;

1. Child and Family focused

2. Health Promotion

3. Transformation

4. Settings

5. Information and Communication

6. Evidence Based and Sustainable

Each principle has an aspirational statement and then indicators to be used to evidence achievement towards a principle. They can be used to assess an existing service or to develop a new service and can be used for a condition across a pathway e.g asthma or for a service e.g. GP practise.

The principles have been developed as a single A5 poster for ease of use and are colourful and visual. A postcard has also been developed which summarises the feedback from young people and families but also translates the principles for families so that they know what they can expect from services. Figures 1, 2, 3.

Local organisations are encouraged to add their own and healthwatch websites to the postcards to allow continuing feedback from families. 


\section{What do children, young people and families want fiom hallth services?}

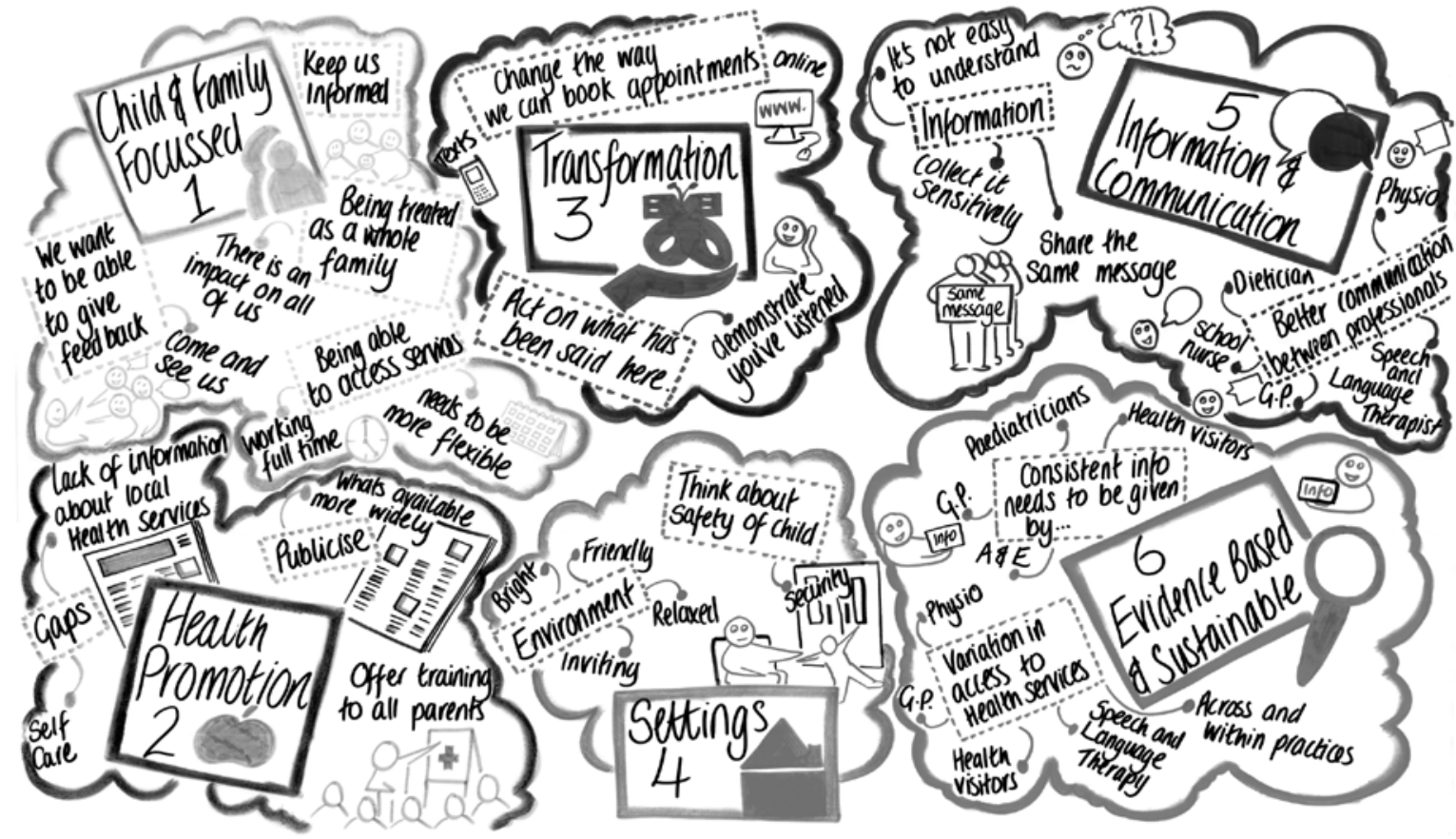

Abstract G32 Figure 2

Principles for commissioning and delivering better health outcomes and experiences for children and young people so that they are comparable with the best in the world.

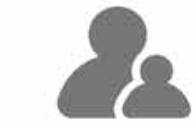

PRINCIPLE 1

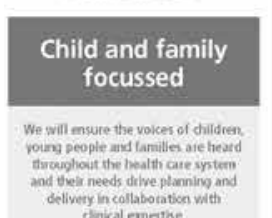

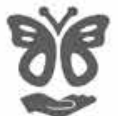

PRINCIPLE 3

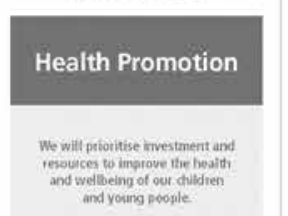

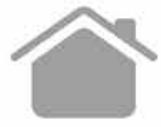

PRINCIPLE 4

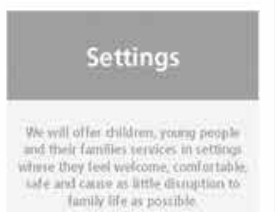

Strategic Network for Child Health and Wellbeing in the East of England

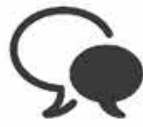

PRINCIPLE 5

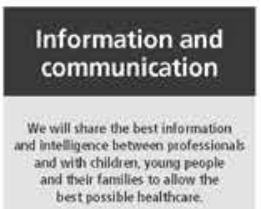

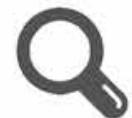

PRINCIPLE 6

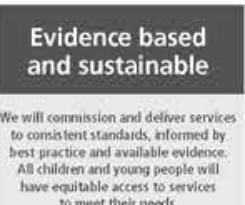

Evidence based and sustainable to meet their needs
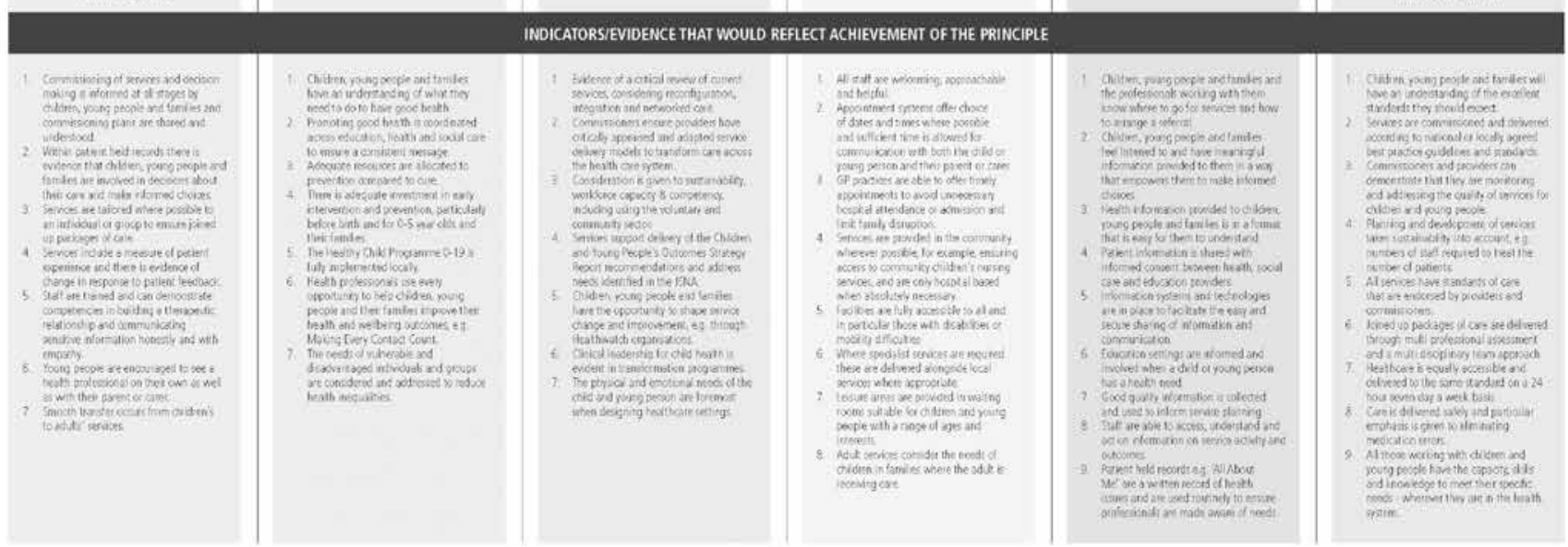
Conclusions The principles define a common language and shared sense of purpose for professionals and families and can be used as a platform of small or large scale change and improvement. They compliment the NHS mandate and the children's and young person' s outcome report and sit at the centre of the NHS change model. They are easy to use and flexible and can be used nationally and easily adapted for other services.

\section{G33 CHILDREN WHO DID NOT ATTEND (DNA) COMMUNITY PAEDIATRIC CLINICS. DEMOGRAPHICS AND FOLLOW UP OUTCOME}

doi:10.1136/archdischild-2013-304107.046

CL Furnell, F Finlay. Child Health, Sirona Care, Bath, UK

Aims Non-attendance at clinic appointments puts children at risk of avoidable ill health. Wasted clinic appointments cost the NHS $£ 700 \mathrm{~m} /$ year with up to 6 million appointments wasted.

The aim of this study was to assess outcomes for children who DNA, following introduction of a red (urgent appointment sent), amber (further routine appointment offered), green (appointment offered only if requested by family) coding system.

Method Records of 100 children who DNA over a 3 month period were reviewed. Information on age, diagnosis, outcome, was recorded and analysed using Microsoft Excel (figure 1).

Results 55 DNA's were classified green, 43 amber and 2 red (figure 1). Diagnosis was variable: $40 \% \mathrm{ADHD}, 12 \% \mathrm{ASD}, 9 \%$ behaviour and $9 \%$ developmental delay

Green 55: 10 new, 45 follow up.

30 did not request further appointment, 25 requested a further appointment - 24 subsequently attended, 1 DNA for a second time.

Therefore 30 appointments were 'saved' - parents did not request further appointments. Before the red, amber, green system was introduced these families would have received another appointment.

Amber 43: 2 new, 41 follow ups.

28 subsequently attended, 15 DNA'd again.

Of these 15 , on reclassification:

8 green (5 ADHD, 1 attachment, 1 anxiety, 1 ASD), 1 parent requested a further appointment and 7 did not.
6 amber, (5 had ADHD and 1 sleep difficulties). 4 subsequently DNA again and 2 attended.

$1 \mathrm{red}$ (developmental delay). Attended the next appointment.

Red 2 (child protection plans), attended subsequent appointment.

Discussion Out of 100 DNAs 30 appointments were saved as patients classified as green did not request a further appointment. The number of DNAs per year in our department (2011-2012) was 905 of 7320 appointments. Extrapolating further we are potentially saving 271 appointment slots per year (45 clinics) compared to our previous system where all DNAs were automatically offered a further appointment.

Of 43 "amber" children, 28 attended the appointment sent, 15 DNA again. Some were subsequently classified as green, those with $\mathrm{ADHD}$ were assumed to be no longer on medication.

The 3 "red" children all attended further appointments.

\section{George Still Forum}

\section{G34 IS THERE A LINK BETWEEN ATTENTION DEFICIT HYPERACTIVITY DISORDER (ADHD) AND DELAYED PUBERTY?}

doi:10.1136/archdischild-2013-304107.047

D I Kevitiyagala, F Finlay. Community Child Health, Sirona Care and Health, Bath, UK

Introduction Recently, our department has seen 4 boys with $\mathrm{ADHD}$ present with delayed puberty. Only one was on treatment for ADHD with no effect on growth. We conducted a literature search to discover if there was a documented link between ADHD and delayed puberty. The full results will be shared during the presentation but a brief summary is below.

Methodology A PubMed search using the terms "ADHD" [Mesh] AND "delayed puberty" found only one relevant article out of three identified. An OVID Medline and "Google Scholar" search revealed three further relevant articles.

Discussion Most of the literature focuses on the link between $\mathrm{ADHD}$ and delayed growth in the presence of stimulant medication. Few papers look also at the effect of either ADHD or stimulant medication on puberty directly. Out of the four papers found, there were conflicting opinions.

\section{DNA's}

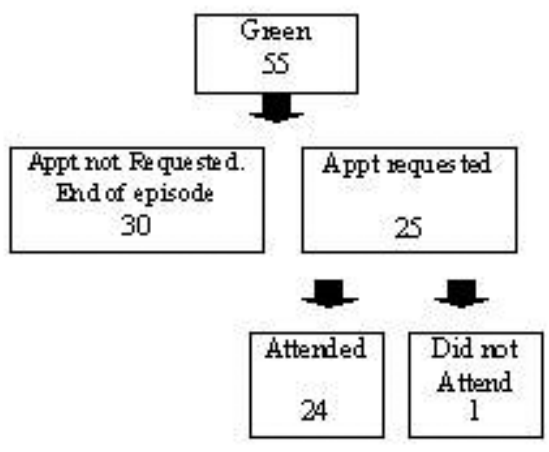

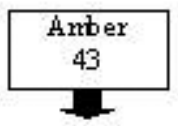

\section{Offered Appointment} Appointment

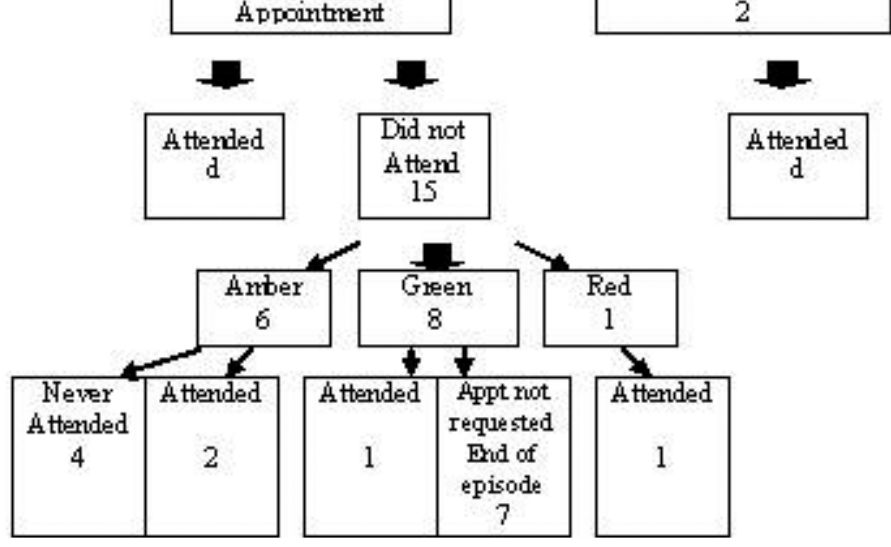

